\title{
Cost/Performance: A Business Discipline
}

\section{Charles Caldwell}

is CEO of NextQuarter LLC and a business process reengineering, automation and asset management authority with $25+$ years experience working with clients such as AT\&T, EDS, Exxon/Mobil, Fiat, Ford, HP, IBM, Microsoft, Union Carbide, US Surgical and hundreds of solution providers and professional services firms. He specializes in benchmarking best demonstrated practices, designing semantic tools and developing interactive platforms to improve Cost/Performance. He is chief architect of NextQuarter's knowledgebase of econometrics, models, frameworks and methodologies used to assess applications, practices, cycle times/ROI and core competencies of thousands of corporations, solution providers and professional services firms to enhance value creation and Cost/Performance.

Keywords: ROI, corporate governance, cost performance, management, mastery

Abstract Are you prepared to address transformational challenges that are impacting business today? Do you have fresh perspective about solutions and best practices? The world has changed. Business challenges are more complex. Events and situations that shift markets and demand happen quickly with little or no warning and create strategic opportunities for those who are prepared. Current approaches to problem solving often lack perspective on key issues confronting people, societies, government and business. Answers are often rooted in new ways of thinking that change perspective.

Cost/Performance is a discipline (low cost - high performance) designed to improve agility and responsiveness while minimizing risk and exposure. Do you have an effective process for transforming business? Do you have discipline to achieve superior Cost/Performance? This paper introduces concepts, models, frameworks and roadmaps developed from years of intensive research. Insight and know-how presented focuses on changing thinking about talent, practices and capabilities. Consider possibilities created by rethinking investments when greater emphasis is focused on examining business fundamentals and interrelationships between industries, markets, employees, products, services, technologies, systems and states and conditions of practices. Journal of Digital Asset Management (2006) 2, 303-314. doi:10.1057/palgrave.dam.3650051

\section{EXPECT THE UNEXPECTED}

Approximately $\$ 6.2$ trillion of the world's annual economic output in 2005 was wasted or attributed to fraud and abuse. Capturing 1 percent of these funds annually would generate over $\$ 60 \mathrm{bn}$ in new revenue to improve world economies (Figure 1).

Business dynamics are shifting. Compliance mandates, high energy prices, natural disasters, terrorism and virtual warfare are transforming business. Adjusting to a world that never stops is demanding. Agility and responsiveness are synonymous with change and chaos. It is hard to manage change, but almost impossible to react to chaos without the right perspective.

Chaos introduces fear, uncertainty and paralysis. Relationships, systems and corporate silos represent barriers to Cost/Performance.
Inability to respond to certain situations and events leads to problems and missed opportunities.

Organizations that embrace change find strength and conviction to reclaim competitive positioning. Paradox lies in perception and structure. Altering perceptions and breaking down barriers changes perspective and outlook.

Cost/Performance induces transformation and creates discipline to enable step change. Cost/ Performance supported by ROI analysis and quick delivery represents the new paradigm for business. Transformation is a core competency that enhances value creation while limiting risk and exposure.

Cost/Performance is a business goal/objective that focuses on obtaining the best Cost/ Performance ratio possible. Corporate 


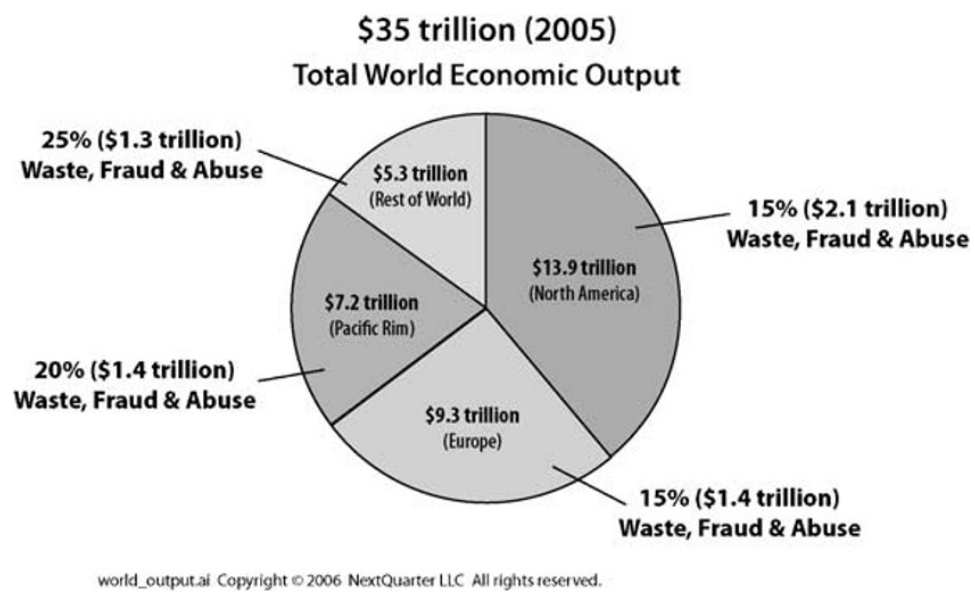

Figure 1: Impact of Waste, Fraud and Abuse

stakeholders are direct beneficiaries of Cost/ Performance directives. Value creation is a function of Cost/Performance. Stakeholder Cost/Performance benefits are greatest when corporate resources are balanced and aligned.

Ability to overcome business challenges requires some degree of mastery. Transformation, environmentalization, personalization, normalization and realization represent core competencies that enable organizations to respond to change. Cost/Performance is a discipline (successive process) designed to guide strategic investments and align and position resources to meet goals and objectives.

\section{GOVERNANCE AND COMPLIANCE}

In 2005, governance and compliance practices impacted over $\$ 3.4$ trillion in US spending 28 percent of US GDP. Over $\$ 1.1$ trillion in US corporate expenditures (9 percent of US GDP) in 2005 was hidden in overhead. Hidden costs are difficult to measure, and even harder to control. Costs are hidden in people and information (Figure 2).

The governance and compliance market revealed itself overnight in the form of a regulatory response to corporate fraud and abuse. Many corporations have become keenly aware of mandates created by Sarbanes-Oxley, Bank Secrecy Act, Basel II, HIPAA, Check 21, the USA Patriot Act and other legislation. Few people, however, fully understand how governance and compliance practices and strategic capabilities impact Cost/Performance.
Governance and compliance has little to do with technology and everything to do with people. Identify the needs and interests of people and it is easier to solve problems and create opportunities. It is also important to recognize that problems cannot be solved on the level they were created. The challenge is perceptions of problems vary and self-serving interests get in the way of business.

Governance relates to the act or process of focusing on doing the right things. Compliance is the act of complying with a request, demand or common law. Together they interrelate to support how we live and work. People are challenged by information and technology. Personal bandwidth is at an all time low and the pace of business never slows down. As a result, it is difficult to keep up. Automation creates more time to focus on important work.

A key challenge is learning how to maintain universal understanding of policies, controls, capabilities and procedures needed to support business when they are constantly changing. There are no quick solutions - only possibilities. Effort needs to focus on making more people part of the solution. The first step is to gain a better understanding of knowledge worker Cost/Performance.

\section{TRANSFORMATION}

Change comes in small increments and through seismic events. Chaos tends to have indirect effect. What appears to be an unrelated event 


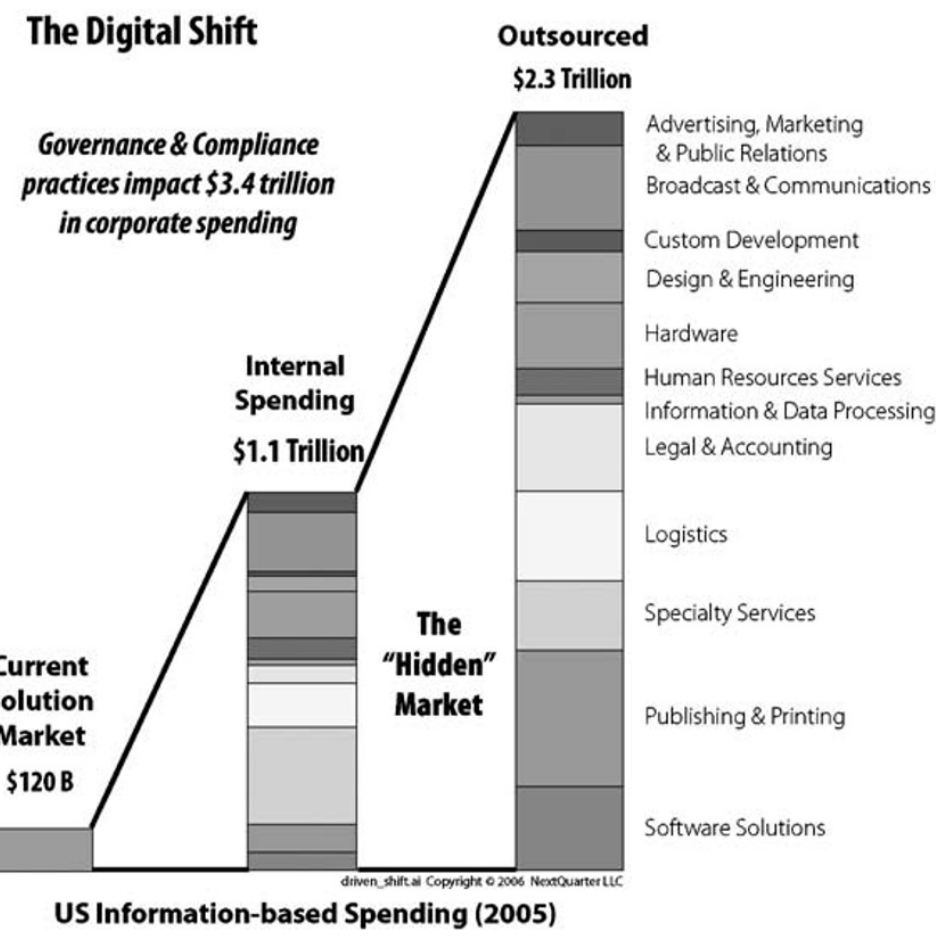

Figure 2: Hidden Costs - Insourced vs. Outsourced

\section{Successive Stages of Cost/Performance Process}

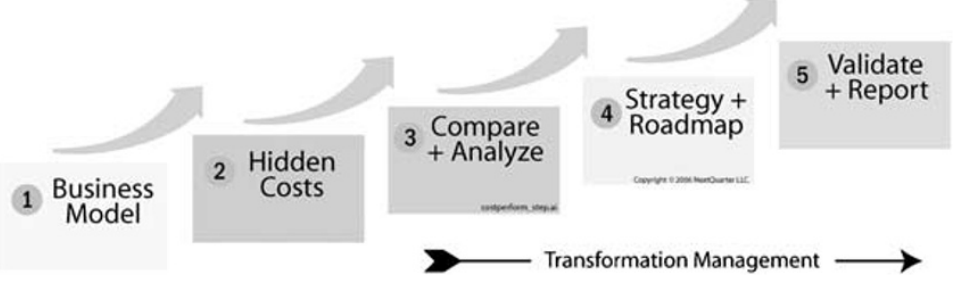

Figure 3: Cost/Performance Management

may trigger an opposing force that can reverberate through a society. Transformational forces are often acute and driven by growth of networks and populations.

Many situations and events that appear separate are actually interlinked and cause fluctuations in economic, environmental and cultural conditions. A catastrophic event on one side of the world can cause instantaneous effect in another. Chaos theory and the butterfly/ domino effect explain this phenomenon. A classic example would be the situation created by oil and auto producers. Consumers have little or no control over fuel prices, vehicle efficiencies and the green house effect.
Transformation is ruled by laws of cause and effect. Operating in a vacuum is not only risky, but potentially life threatening. Cause and effect introduce dynamics that create imbalance. Perspective plays an important role in determining how people will react under certain states and conditions. Cost/Performance measurement can help to gauge perspective. Points of view change when perspective swings too far in one direction. It is important to know tipping points and their implications.

Transformational forces put Cost/Performance in motion. Responsiveness is a function of cause and effect. Myopic perspective hinders abilities to respond to the needs of others. Cause and 
effect create exposure for those who fail to predict outcomes.

Cost/Performance represents a way of doing business. Stakeholder engagement is a function of understanding and satisfying collective needs and interests (Figure 3).

The model above illustrates how successive stages of development are continuously leveraged to support learning. The evolutionary nature of the process builds upon success factors that support value creation.

Business modeling sets up opportunities to understand interrelationships. Process analysis and knowledge worker profiling creates opportunities to identify and measure hidden costs. Comparative analysis introduces opportunities to learn from early adopters who have mastered best practices and leveraged new capabilities. Each stage produces insight and know-how to develop strategy and craft roadmaps to guide Cost/Performance. As cost efficiencies and performance improve to support value creation, capabilities and procedures needed to validate effectiveness and enhance reporting become a natural part of doing business.

\section{CORRECTIVE MEASURES}

The world economy is complex and influenced by the reactive practices of governments and multinational corporations. Self-serving interests, nonstop commerce, technological advancements and the endless pursuit of growth and profits have increased adversity and conflict. Take a close look at economic states and business conditions and you will see that the world's ecosystem has become unbalanced.

Each day, we are constantly reminded of the heightened intensity of terrorist events, corruption and environmental disasters that are changing our world. Increasingly, we are forced to take glimpses of states and conditions that are destabilizing our way of life. As a result, there is a growing sense of uneasiness building within more and more people.

Each year, business structures and interrelationships are becoming harder to manage. This is due in part to the:

- evolutionary characteristics of technology;

- need to reduce costs and improve productivity;

- indecisive response to events and situations;

- nature of vertical and horizontal applications;

- change in businesses, industries and markets.

Decision making is most effective when people are well informed to make the right decisions. Today, the ability to make the right decisions often requires having access to real-time information. The challenge is how to sift through mounds of data to find the information you need. Situations are constantly changing, which means challenges keep getting more complex (Figure 4).

Managing through heightened periods of uncertainty is difficult. This is why it is important to be proactive. Organizations overcome adversity by reducing fear of the unknown. Anticipatory practices help to reduce

\section{It is important to "take time out" to understand the consequences of our actions.}

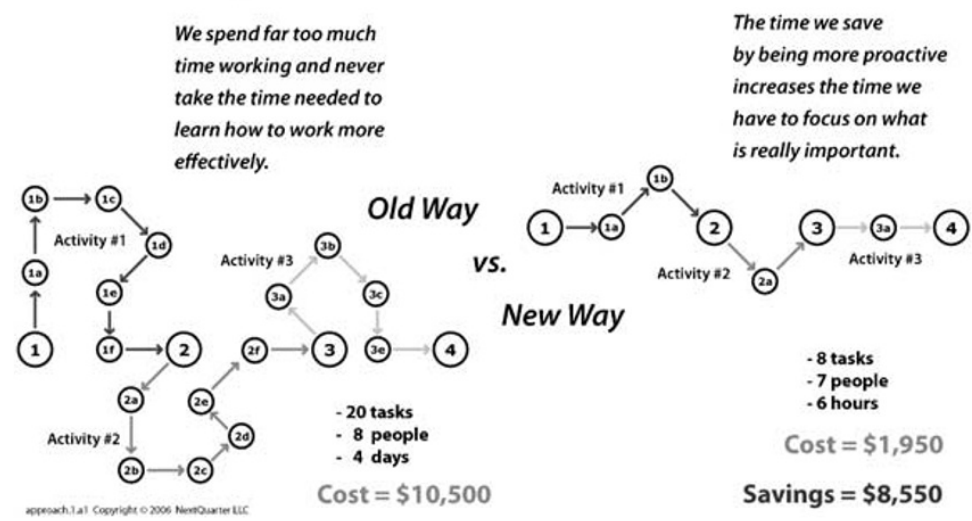

Figure 4: Effective Business Process Management 
the unknown by enhancing levels of awareness. An organization's ability to manage adversity is predicated on the existence, quality and use of anticipatory practices. Reactionary management styles enhance risk and reduce Cost/Performance.

Human beings have the tendency to react to situations without fully understanding the consequences of their actions. As a result, organizations get into compromising situations - as the pace of business increases, so do instances of problems. This is one reason why so many issues are surfacing today. Without proper safeguards, the reactionary nature of people gets in the way of doing business. Operate in internet time and the problem gets easier to understand, but more difficult to manage.

\section{COLLECTIVE GOVERNANCE}

Defy universal laws of cause and effect and you create imbalance. The answers to our problems are basic, but collective. It is time to address these problems. We have solutions, but for them to work we must enhance awareness of common concepts and principles.

People learn when they are engaged and empowered. People, however, must be enticed to learn. Reward is focused on making money. Learning is an afterthought, which is partly why our education systems are in turmoil. It is important to reward success, but within reason. The issue is how we define success. Reward systems at Enron, Tyco and MCI created opportunities for executives to bend the rules. Change our reward systems to focus on creating value for all stakeholders, and we start to solve some of our problems.
To some degree, reward systems fail to recognize good governance practices. The world needs effective leaders. Unfortunately, they are in short supply. Today, leadership is measured more by how much you make than by what you do. Greater emphasis needs to be placed on getting people to do the right things. Praise good governance and the way we measure success, and we begin to change our systems. Everyone benefits when we improve the quality of governance practices.

Effective leaders practice what they preach. Character and passion found in effective leaders is also present in the people they lead. The issue is our concept of governance. Corporate structures and management practices that appeared to work in the past have become obsolete (Figure 5).

Governance begins with one person trying to do the right things. Somewhere in time, we neglected to recognize basic rules of governance. The problems we need to overcome can only be solved collectively. Failure to recognize this fact makes all other points irrelevant. As human beings, we exercised our "free will" and neglected to live within the confines of cause and effect. As a result, our world has become unbalanced.

To a large degree, directives must come from governments and corporations. But in the end, it comes down to people. We can continue down this unsustainable path or we can introduce corrective measures. This will require a shift in thinking. This is why governance and compliance is so important. Initially, we need to focus on improving governance practices

\section{Be Prepared - Anticipate the Possibilities}

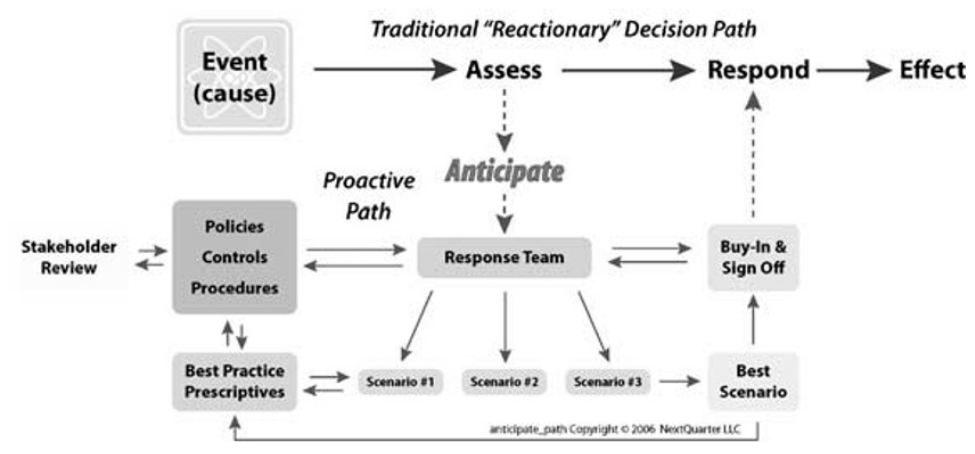

Figure 5: Anticipate the Inevitable - Cause and Effect 
so we can begin to create some degree of balance.

Governance practices are supported by policies, controls and procedures that are needed to guide work efforts. Compliance mandates are defined by rules, regulations and standards that are established to set parameters and expectations for meeting requirements. Together, governance and compliance interrelate to create a symbiotic framework to support the way collective resources and efforts align to ensure people focus on doing the right things.

\section{DISCIPLINE}

Organizational effectiveness increases when collective efforts align to support a common business model. Cost/Performance results are based on sustainability factors and abilities to keep people engaged and focused (Figure 6).

\section{Perspective}

Reactionary practices increase volatility and lower Cost/Performance. Gaining a keen understanding of corporate interrelationships creates opportunities to improve the quality of management practices. Business models present the context in which structure and relationships are measured. Perspective provides insight needed to optimize practices.

\section{Hidden Costs}

It is difficult to justify practices without good Cost/Performance data. Many costs are hidden. Outsourcing increases levels of uncertainty and reduces opportunities to fine tune practices. Activity-based metrics are used to measure costs to identify deficiencies in practices. It is dangerous to operate blindly when it is possible to analyze and control these costs.

\section{Comparative Analysis}

Early adopter research provides insight into success factors to enhance learning. Best demonstrated practices of early adopters who have leveraged strategic capabilities help introduce step change and direction on how to improve Cost/Performance.

\section{Strategic Roadmaps}

Insight gained to improve Cost/Performance must be put to use. Where do you start? Where do you focus? The answer is to map out how specific capabilities can be used to reduce costs. ROI calculations and strategy combine to guide development and deployment efforts.

\section{Validate and Report}

How well are you performing? Use the opportunity to tell your story. Engage stakeholders and have them tell you how you are performing. Use third-party specialists to

\section{Cost/Performance Management Process}

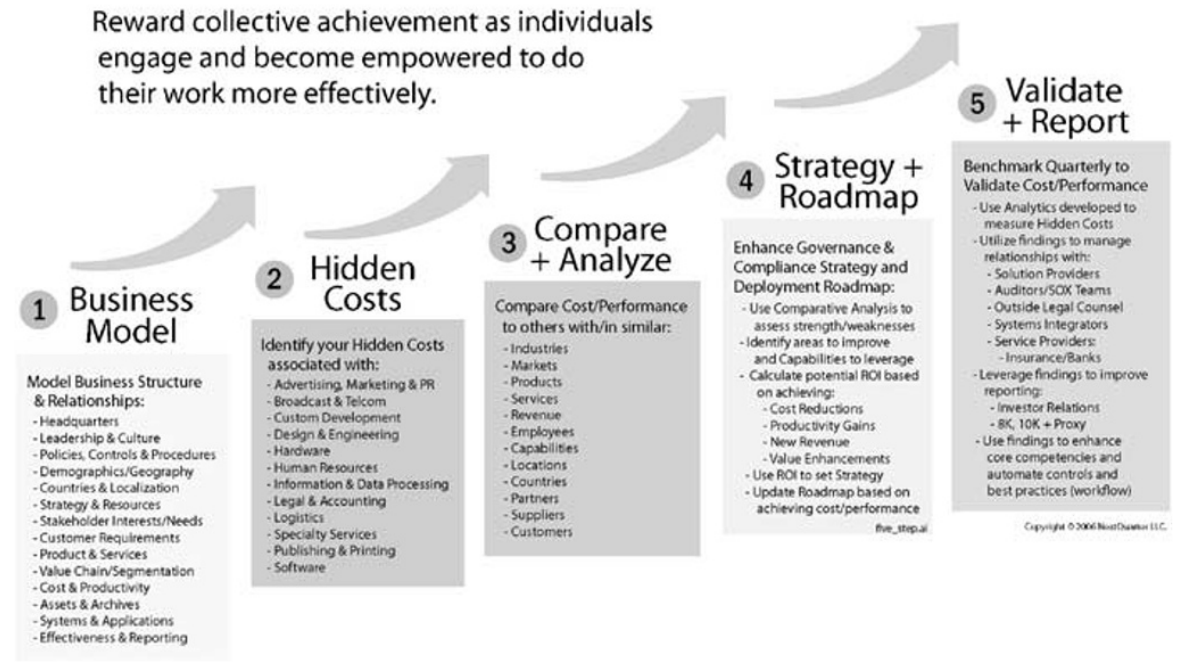

Figure 6: Myopic Perspective Hinclers Responsiveness 


\section{Solution Capability Positioning}

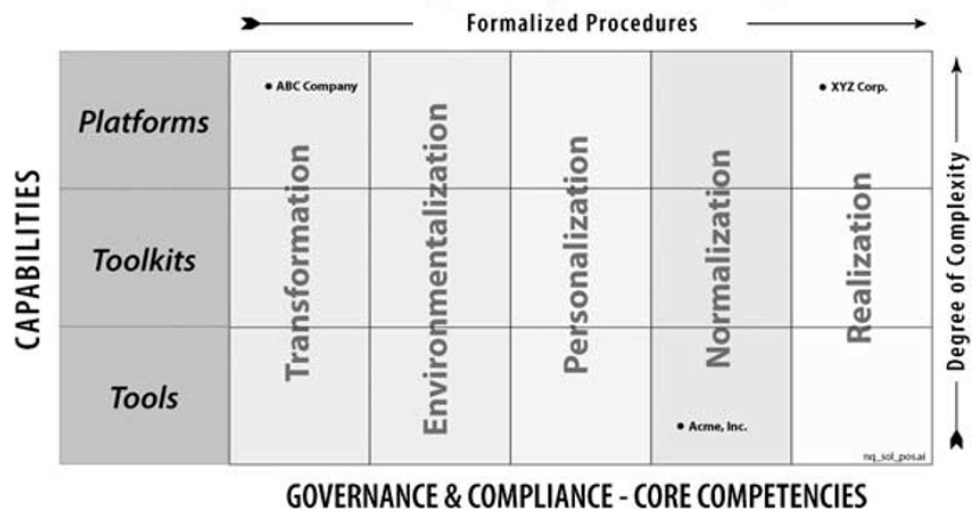

Figure 7: Ability Requires Some Degree of Mastery

validate the effectiveness of policies, controls and procedures.

\section{MASTERY}

The ability to enhance business practices is contingent upon mastering several core competencies (Figure 7).

\section{Transformation}

Step change is a transformational process that enables organizations to make significant advances without having to disrupt operations. Corporate policies, controls and procedures, and regulatory requirements and standards impact cost performance. Step change involves engaging knowledge workers and capabilities in efforts to identify and reduce hidden costs. Transformation is an evolutionary process designed to optimize effectiveness.

\section{Environmentalization}

Business modeling involves mapping interrelationships between people, workflow and systems. Legislation, enforcement, industry presence, market focus and technologies influence the way people work. To adapt to change, predictive and detective controls are used to manage risk. Corporate culture and operating environments influence abilities to enhance and maintain Cost/Performance.

\section{Personalization}

Ability to recognize, manage and protect personal information and assets is dependent on strategic capabilities. Strategy influences how people are empowered and capabilities are leveraged to support value creation. Outsourcing can compromise data and reduce opportunities to optimize practices. Financial, health and identity-based data must be managed effectively to maintain integrity of sensitive information and guard against misuse.

\section{Normalization}

Semantic models, ontologies, taxonomies and databases combine to manage information. Data must be normalized to reduce the complexity of code needed to support business operations. Metadata (data about data) standards enable organizations to configure disparate systems and knowledge. Normalization enables organizations to reduce redundancies while enhancing the integrity and flow of information.

\section{Realization}

Practices, activities and/or tasks that are sanctioned must be documented to meet attestation audits. Proof of occurrence and the quality of detective and corrective measures provide insight into the effectiveness of control design. Efforts made to evaluate/test design effectiveness through inquiries, observations, walkthroughs and document inspections, combined with a thorough risk assessment provide evidence needed to satisfy regulatory and stakeholder requirements.

\section{ENGAGED AND EMPOWERED}

All organizations must strive to create balance and unity. This is difficult when organizations 
outsource business practices. Great innovation occurs when people come together to share and express ideas. Working towards a common vision creates opportunities for people to align to meet an objective. Problems arise when needs and interests are going in different directions.

Outsourcing can damage morale and heighten levels of insecurity when employees feel their jobs are at risk. The needs of "internal" employees and "external" consultants can vary significantly. This is due to conflicting interests and objectives of different reward systems. Competitive work environments introduce conditions that produce ulterior motives that destabilize workgroups.

Corporate environments are complex, especially when you consider relationships that exist between different stakeholders (employees, customers, partners and investors). In an ideal world, stakeholder needs and interests align to meet corporate goals and objectives. In reality, this is not possible given the dynamic nature of relationships. This is why it is so important to model and assess interrelationships (Figure 8).

Gaining perspective of individual needs, interests and expectations creates opportunity to model states and conditions that impact business conditions. With the right tools and metrics it is possible to balance internal and external resources to meet a common objective. Policies, controls and procedures should be established to manage resources based on Cost/Performance benchmarks. Reward systems should be designed to ensure collective efforts align to meet Cost/Performance objectives. Individuals are rewarded based on collective results to ensure group efforts are optimized. Key success factors:

\section{Receptivity}

Opening to change, promotes learning.

Receptivity is an important trait that is reflected in culture and adaptability characteristics. Executives who promote learning enable transformation to revitalize business.

\section{Proactivity}

Anticipatory practices create opportunities to respond more decisively to activities and events that shape business. Concerted effort to enhance decision making helps people focus on doing the right things.

\section{Responsiveness}

Organizations that are prepared to respond to shifts in business and market conditions focus on anticipating change. Making sure the right people are in the right jobs with the right resources strengthens abilities to respond to the needs and interests of people who can make a difference.

\section{Validation}

Proving effectiveness puts credibility behind everything you do. The journey is as important

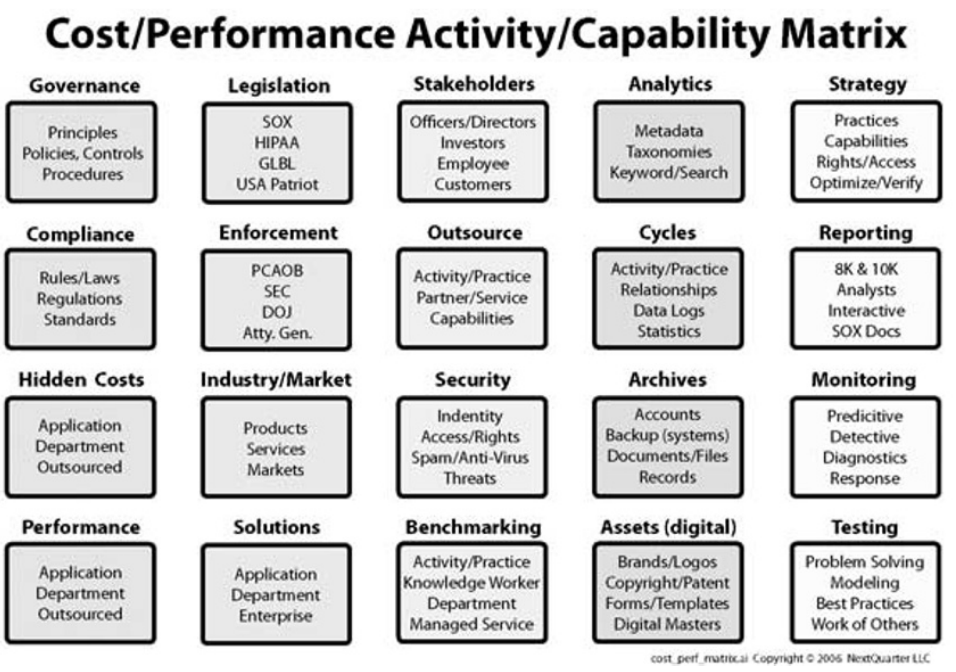

Figure 8: Core Competencies - Cost/Performance mix 
as a successful outcome, but can tell the story. If storytelling is an afterthought, so are the benefits of the memories - capture the experience before it is lost in time.

\section{COST/PERFORMANCE DIRECTIVES}

View from the top sets the stage for delivering value. Vision and focus provide direction for people to follow. Ethical and moral judgment is the result of trust and integrity that exists in and between people. Cost/Performance provides insight into how well people and resources align to deliver value. As a rule, the greater the Cost/Performance ratio (low cost/high performance), the more people are focused on doing the right things.

Leadership is measured based on the Cost/ Performance that exists in people. Effective leadership is found in organizations where people are engaged and empowered. Effective leaders put the needs and interests of their people first. People want to be lead. To become fully engaged, however, they must believe their needs and interests are recognized and supported, and also know they are doing the right things.

Cost/Performance directives outlined below were developed to raise corporate standards and levels of awareness of knowledge required and best practices to meet the demands of the 21st century. Cost/Performance modeling introduces a logical approach for implementing best practice prescriptives while measuring states and conditions to improve effectiveness.

It is rare to find good examples of organizations that have optimized Cost/ Performance. This is primarily due to the way organizations have evolved over the past 40 years. Ability to develop and maintain "end-toend" solutions is almost impossible today due to the configuration of business and system structures. To meet the responsive demands, organizations must re-examine business fundamentals while implementing step change (Figure 9).

Cost/Performance directives presented below were developed assessing best practices, lifecycles, technologies, market drivers and trends. These directives are the result of a five-year intensive research effort. To maximize Cost/Performance,
Key Business Challenges

(Transformation Period: 2006 - 2011)

-Make, Buy or Outsource Assessment

- Retention/Engagement of Top Talent

- Lifecycle Design for Reuse/Renewal

-Enhanced Agility \& Responsiveness

- Security \& Event Simulation Practices

-Shift from Cost to Asset Generation

- Breaking Down Corporate Silos

- Change to Anticipatory Management

-Social/Environmental Responsibility

key_ctulungeas Copright 02006 Natouster UC

Figure 9: Overcoming Key Business Challenges

organizations must overcome the following challenges:

\section{Overextended}

People are working too hard. Focus and effort are overshadowed by voice mail, e-mail and meetings. Work weeks keep getting longer, Cost/Performance is declining and family and community are suffering.

\section{Hidden Costs}

Corporations are outsourcing their strategic advantage. Data needed to cost justify practices and optimize capabilities are often outsourced. Hidden costs are not recognized and data needed to manage services are not available and/or utilized.

\section{Step Change}

Inability to change with the times is leaving people behind. Learning must be emphasized, practices updated and capabilities made less burdensome. Cause and affect analysis should be used to advance efforts and change beliefs.

\section{Strategic Cycles}

It is not enough to establish policies, controls and procedures; they must be practiced to be effective. The way people are organized and positioned, when decisions are made, and how projects interrelate is strategic. 


\section{Effectiveness}

People who need to be engaged are often not recognized. Those who are engaged may not be effective or do not know important characteristics about the business. Capabilities that could be leveraged may be overlooked or misunderstood.

\section{PROBLEM DEFINITION}

So where do you start? How do you start a Cost/Performance program? The answer is to focus on problem definition. Billions of dollars are wasted each year on projects that fail. Projects fail for various reasons:

- Poor design/definition;

- Change in focus;

- Scale/Scope;

- Cost (over budget);

- Mismanagement

- Poor buy-in.

Failure is inherent in approach and perspective. There are clearly effective and ineffective ways to solve business problems. Projects begin and end based on how problems are defined. Problem definition involves understanding cause and effect. It is vital to account for the unknown. This is why econometric data models, roadmaps, frameworks and strategy are so important.

It is not uncommon for people to act first and deal with consequences later. Not enough time and effort are given to problem definition and too much on building solutions.

Best Practice $=$ Understand the problem first and the solution will present itself.

It is not enough to have good intentions; problems must be identified and modeled to be understood. Problem definition is an art and a science. Models create opportunities to enhance results. The objective is to see what needs to be addressed. Gaining a strong understanding of a problem is hard work, but vital to achieving a successful outcome.

Hidden costs and unforeseen events/situations cause problems to grow exponentially. One problem often leads to another. Over time, scale and scope can expand/contract based on states and conditions. To account for change you must anticipate change.

Best Practice $=$ Integrate learning into the problem definition process.
Business problem definition begins by examining interrelationships that exist between people, workflow and systems. Trust and integrity enable people, processes and solutions to coexist. Trust is based on commitment and truth. Integrity is a measure of how well core competencies are mastered.

\section{Approach}

Integrity requires people to remain true to their beliefs and convictions while adhering to a moral/ethical code of conduct. Breaking trust introduces fear and uncertainty that can damage even the best relationships. When people are true to their beliefs, personal pride and passion is visible in the quality of people's work. There are two approaches to problem definition:

(1) Short term (reactionary).

(2) Long term (anticipatory).

It is not always possible to take the long-term approach because of time and resources. But the long-term approach is always best. This is why it is so important to think ahead.

Best Practice $=$ Optimize design by accounting for future reuse/renewal.

Short-term gains realized at the expense of long-term interests degrade integrity. Individuals who maintain their sense of integrity set precedence for others to follow. When reactionary measures are necessary, take care in realizing that short-term responses can lead to long-term disasters. During times of change, people fear the unknown. Placing trust in how problems are solved helps to build faith and confidence in future outcomes (Figure 10).

\section{Goal of Effective Business Problem Definition}

- Improving Accountability

- Controlling Hidden Costs

- Reducing Risks \& Liabilities

- Simplifying Complexities

-Balancing Resources

problem_defineai Copyright 02006 NextQuarter LCC

Figure 10: Achieve Substainable Results 
REACTIONARY MANAGEMENT

There is a growing sense of unrest in the world. Living in a period of change, such as the one we are experiencing, creates an underlying sense of uneasiness. Awareness, positioning and extent of personal resources tend to influence how we feel and react. Change is often painful and unsettling, and can have a numbing effect. The danger of withdrawing is living within a false reality (Figure 11).

Change is not something you control; it is a natural part of life. Take your eye off of what is happening around you and the potential impact can be overwhelming. The only way to effectively manage change is to stay in front of it. This is why it is best to live in the moment while always keeping an eye open on the future.

Universal laws of cause and effect influence change. Nothing happens by chance, there is a natural order to everything. What we observe in life is based on an infinite number of unseen events - commonly know as the "butterfly effect." Events happen and/or arrive as a result or consequence of some preceding event. There is continuity between events governed by the laws of cause and effect.

To successfully manage change takes a great deal of hard work. If you do not focus on being at the forefront of change, it becomes difficult to survive. During erratic periods of time, those who survive turn change into an opportunity. Early adopters of change see what others fail to see or refuse to see. It is risky to create the future, but even riskier not to try.

\section{ANTICIPATORY MANAGEMENT}

Organizations that actively participate in creating their own future must be responsive. An organization's ability to respond is based on the

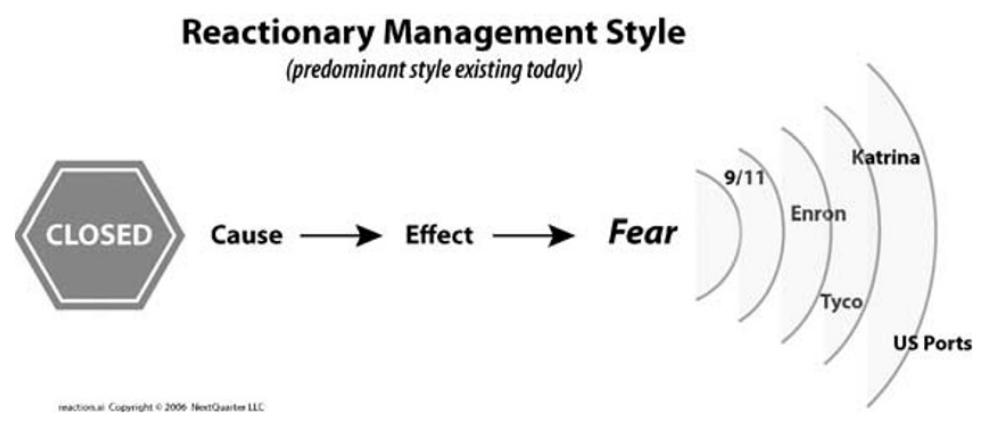

Figure 11: Fear and Paralysis Hinder Responsiveness

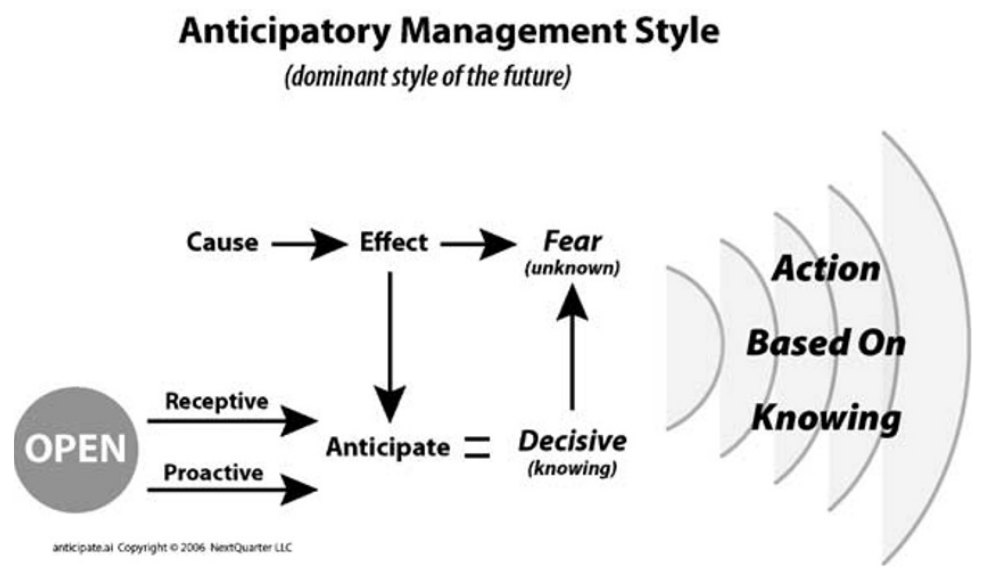

Figure 12: Anticipatory Management - Getting the Right Results. 
collective knowledge and understanding of its people and how they are supported. It is not enough to be responsive; the differentiating factor between success and failure is based on being open (Figure 12).

Adapting to an open way of thinking takes courage. Courageous leaders are not fearless they just take action in spite of their fears. More than 25 years of experience studying knowledge worker behaviors has led NextQuarter analysts to conclude that individuals follow the path of least resistance unless influenced by a higher presence. Shifts in beliefs seldom occur without a dramatic shift in consciousness. The challenge is how to nurture this effect without the influence of shock and awe. Introduce a motivator and people will change, but is the change lasting?

\section{Receptivity}

Corporations that are open to change tend to learn more effectively. Receptivity is an important trait used to assess corporate culture and adaptability characteristics. Organizations that are open to receive new information and ideas are able to adopt better to changes in markets and economic conditions. Learning organizations are more apt to change business practices to enhance performance and create growth opportunities for stakeholders.

\section{Proactive}

Corporations that take the time to anticipate future activities and events are better positioned to respond to problems and opportunities. Organizations that make concerted efforts to enhance decision-making abilities benefit when detective and predictive controls are developed to increase agility. Corporations that have thought through many business scenarios are better positioned to established effective strategies and policies to ensure that employees focus on doing the right things. Proactive corporations are able to address threats and seize opportunities when they are prepared to take action and predict outcomes.

\section{Responsiveness}

Corporations that are open to receive and engage in anticipatory development efforts are best positioned to respond to stakeholders. These types of organizations have strong governance principles and make significant efforts to ensure the right people are in the right jobs with the right resources. Results that these types of organizations are capable of producing become apparent to stakeholders who are served and kept well informed. 